\title{
Application of Autofluorescence for Analysis of Medicinal Plants
}

\author{
Victoria V. Roshchina, Andrei V. Kuchin, and Valerii A. Yashin \\ Laboratory of Microspectral Analysis of Cells and Cellular Systems and Optical Department, Russian Academy of Sciences, \\ Institute of Cell Biophysics, RAS, Institutskaya Str. 3, Pushchino, Moscow Region 142290, Russia \\ Correspondence should be addressed to Victoria V. Roshchina; roshchinavic@mail.ru
}

Received 22 August 2017; Revised 29 September 2017; Accepted 19 October 2017; Published 26 November 2017

Academic Editor: Guillermo Moyna

Copyright ( 2017 Victoria V. Roshchina et al. This is an open access article distributed under the Creative Commons Attribution License, which permits unrestricted use, distribution, and reproduction in any medium, provided the original work is properly cited.

Autofluorescence of secondary compounds contained in plant secretory cells may be applied to the analysis of medicinal plants for pharmacy. Emission and prevailing fluorescent pharmaceuticals have been estimated in several models of species such as Salvia officinalis, Berberis vulgaris, Humulus lupulus, and Matricaria chamomilla, by luminescence microscopy, microspectrofluorimetry, and confocal microscopy.

\section{Introduction}

Autofluorescence of plant secretory cells as a parameter for the characterization of pharmaceutical material has not been used yet. It was took place, perhaps, due to a little attention paid to the emission of many natural individual compounds and components of medicinal herbs. However the analysis of the literature has shown that secondary metabolites of plants, including medicinal ones, may fluoresce in visible spectral region [1-3]. Some information found earlier also was in the book devoted to the thin-layer chromatography of extracts from pharmaceutically valuable plants on silica gel plates where separated compounds have been excited by ultraviolet light [4]. The study of the emission may be of interest for pharmacy, so much so that the new book of medicinal plants for pharmacologists has not included the fluorescent method for the analysis yet and is based on the observation of the microscopic images in transmitting light [5].

Earlier the data dealt with autofluorescence of plant secretory cells and their emission spectra in visible region have been described in reviews $[1,6]$ and special monograph [2], but so far the attention of scientists has not concentrated exactly on medicinal species. As a whole, these publications have demonstrated the use of the self-emission phenomenon in the noninvasive analysis of plant secretory cells without histochemical dyes. The occurrence and location of secondary metabolites within tissues and cells have been shown by fluorescent methods [2]. Recently fluorescent technique also has been applied to plant raw pharmaceutical material containing natural drugs in the secretory structures [7]. Here one has seen the emission of drugs such as alkaloids and anthraquinones prevailed in the samples of Chelidonium majus and Frangula alnus, relatively. In first species secretory cells of laticifers containing alkaloids chelerythrine and sanguinarine have fluoresced in greenyellow, while second species enriched in anthraquinones have demonstrated the emission in orange-red. In complex multicomponent samples, such as species Achillea millefolium, Artemisia absinthium, and Calendula officinalis, the spectral interference appears to take place because both terpenoids and phenols brightly fluoresced in glands and secretory hairs in blue or blue-green.

The purpose of the paper is to consider an expressanalysis of the fluorescence in different parts of medicinal plants by various techniques: luminescence microscopy and its modifications such as microspectrofluorimetry and confocal microscopy. In the fluorescence spectra of the objects pharmacologists will see characteristic maxima that may be used in their practice for preliminary identification of drugs.

\section{Materials and Methods}

2.1. Objects. Objects of the research were fresh herbs of common sage Salvia officinalis L., fam. Lamiaceae, European barberry Berberis vulgaris L., fam. Berberidaceae, hop Humulus 
lupulus L., fam. Cannabaceae, and German camomile (chamomile) Matricaria chamomilla L. fam. Asteraceae that have been collected in summer in meadow and plantations near Oka River. Details of the objects have been observed under stereomicroscope Leica MZ16 A (Germany).

2.2. Images of Fluorescent Cells. Autofluorescence of living cells has been observed and photographed by the microscope on the glass slides at room temperature $20-22^{\circ}$ as described previously [7] using Leica apparatuses (Germany) luminescence microscope Leica DM 6000 B (natural emission colors) and laser-scanning confocal microscope Leica TCS SP-5 (pictures were seen in pseudocolors). The sources of the exciting light were mercury lamp, for visual observation, and laser Argon/2 (wavelengths 405, 458, 477, 488, and $514 \mathrm{~nm}$ ), HeNe1 (543 nm wavelength), and HeNe2 (633 nm wavelength). The registration by confocal microscopy has been carried out in three channels. Besides, for chamomile pollen laser-scanning microscope LSM 510 NLO Carl Zeiss was used with the laser Argon $488 \mathrm{~nm}$. Excitation by lasers at wavelengths of $458,488,543$, and $633 \mathrm{~nm}$ has resulted in the emission fluorescence at $505-630 \mathrm{~nm}, 650-750 \mathrm{~nm}$, and $650-750 \mathrm{~nm}$, respectively. Three photomultipliers can catch the fluorescence, separately or simultaneously, by using the pseudocolor effects. The image analysis has been done with the computer software LSM 510 and Lucida Analyse 5. Pseudocolors were chosen according to the excitation wavelength: blue for $488 \mathrm{~nm}$; green for $543 \mathrm{~nm}$; and red for $633 \mathrm{~nm}$. Layered images of the optical slices along the objects were in their pseudocolors. For the presence of nucleus in hop lupulin fluorescent dye Hoechst 33342 (Serva, USA) was used.

2.3. The Fluorescence Spectra. The emission spectra of intact cells have been recorded and analyzed by microspectrofluorimetry and confocal microscopy. In first case the luminescence microscope was combined with the registration system in microspectrofluorimeter MSF-1 $[8,9]$ made by the Institute of Biological Techniques, Pushchino. The excitation of the emission was by light $360-380 \mathrm{~nm}$. The fluorescence spectra of cells were also recorded with the above-mentioned laserscanning confocal microscope Leica TCS SP-5 (Germany) with laser excitation $405 \mathrm{~nm}$. Individual cells or parts of the secretory structures were first identified as a region of interest (shortly ROI) and marked by rings or rectangles. The fluorescence intensity was measured by the emission from the ROI.

The emission spectra of the water and ethanol extracts from $1 \mathrm{~g}$ of studied material, secretory cells or solution of individual compound berberine (Sigma-Aldrich, United States), were recorded by spectrofluorometer Perkin Elmer $350 \mathrm{MPF}-44 \mathrm{~B}$ (UK) in 1 or $0.5 \mathrm{~cm}$ cuvettes.

\section{Results and Discussion}

The observation of autofluorescence may be useful in order to determine variability in the secretory structures fulfilled with drugs and even for the identification of a prevailing component in the sample if it fluoresces.
3.1. Variability in the Fluorescence in Fresh Raw Materials. The color and spectra of the autofluorescence are changed during the plant development [2], and the readiness of raw pharmaceutical material may be well-seen on the images and fluorescence spectra. Similar example for our study was the sample of flowers from common sage Salvia officinalis L. (family Lamiaceae). This medicinal species contains most active medicinal components against diabetes and inflammation [10-14]. Some chemical compounds such as flavonoids, terpenoids, and essential oils are met here and may demonstrate antidiabetic and anti-inflammatory features. As shown in our experiments common fluorescence of the sage flower petal and its secretory hairs has been well-seen under luminescence microscope (Figure 1(a)). At expressanalysis, changing the wavelength of the excitation, pharmacologists may observe the flavonoid blue emission in ultraviolet (340-380 nm), quenching in the hairs under violet light (355-425 nm). The petal of S. officinalis contains a lot of blue color anthocyanins emitted also in blue spectral region. The green fluorescence $(510-520 \mathrm{~nm})$ excited by light $488 \mathrm{~nm}$ appears to link with flavins, with yellow lightening at the excitation in region $450-490 \mathrm{~nm}$, with carotenoids and some anthocyanins [2]. At last bright red fluorescence at the excitation in the range $515-560 \mathrm{~nm}$ belongs to chlorophyll that is usually masked by anthocyanins and seen only under luminescence microscope.

Using confocal microscopy we studied the fluorescence and the emission spectra of secretory hairs enriched in various drugs (Figures 1(b) and 1(c)). There were a lot of different maxima in their fluorescence spectra (Figure 1(c)) that witness the occurrence of different substances in the cells studied. In spectrum of the ROI number 3 the emission was in blue that may relate to terpenoids fluoresced with maxima at $420-430 \mathrm{~nm}$ [2]. The samples numbers 1 and 2 had two maxima, one in blue-green at 490-500 $\mathrm{nm}$ and second in red at $680 \mathrm{~nm}$ that shows the presence of chlorophyll in the secretory cell. Besides the pigment, hair ROI number 4 had big maximum $520 \mathrm{~nm}$ (perhaps, belonging to flavins). In sample ROI number 5, with lack of chlorophyll, there was peak in green only peculiar to flavins and coumarin esculetin [2].

3.2. Prevailing Fluorescent Components in the Samples and New Perspective Organs as Pharmaceutical Material. The task to analyze pharmacological material seems to be dissolved better if the prevailing components are known. As shown earlier [7], similar examples were seen in anthraquinones or alkaloids-containing plants. In this paper we studied alkaloidcontaining species of European barberry Berberis vulgaris from family Berberidaceae that contains more than 30 alkaloids $[10,11,15]$ as well as 10 various phenolic compounds [11, 16]. Alkaloid berberine is prevailing active medicinal agent. The extracts from the roots and, in lesser degree, the leaves of the species and individual alkaloid berberine itself may have anticancer, anti-inflammatory, antioxidant, antidiabetic, antibacterial, analgesic, antinociceptive, and hepatoprotective effects $[15,16]$. It is widely used against cholecystitis, chronic hepatitis, and the infection of postpuerperal period [11]. In traditional medicine fruit and flowers have not been used for pharmacy yet. To keep in mind this perspective 

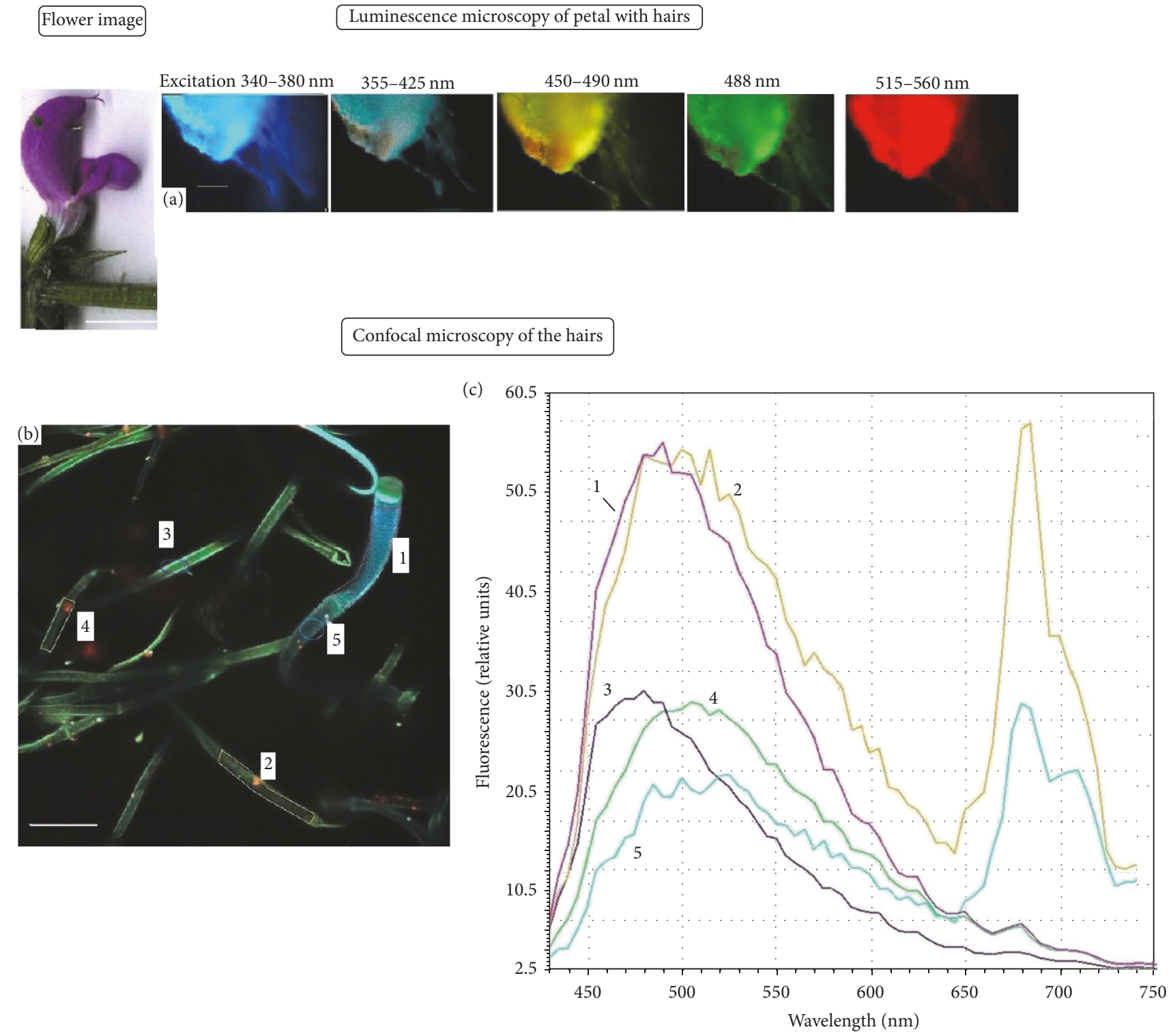

FIGURE 1: The fluorescence of phenol- and terpenoid-enriched secretory cells of flower from Salvia officinalis L. (a) Part of petal with hairs emitted under various excitation of luminescence microscope Leica DM $6000 \mathrm{~B}$, bar $=30 \mu \mathrm{m}$; ((b) and (c)) numbered hair cells of petals fluorescing under laser-scanning confocal microscope Leica TCS SP-5 (laser excitation $405 \mathrm{~nm}$ ) in green-yellow $(\mathrm{bar}=30 \mu \mathrm{m})$.

we have studied these organs by various luminescence techniques. As shown in Figure 2 the contribution of prevailing fluorescent component, alkaloid berberine, was observed under various modifications of luminescence microscopy, from simple luminescence microscope and microspectrofluorimetry to confocal microscopy. At excitation by laser $405 \mathrm{~nm}$ of confocal microscope the surface of fruit containing secretory cells has fluoresced in yellow (Figures 2(a) and 2(b)). The lightening was especially bright in the drop of secretion (Figure 2(c)). In the fluorescence spectra (Figure 2(d)) of both secretory cell (Figure 2(b)) and the secretion drop released (Figure 2(c)) there was maximum $540 \mathrm{~nm}$, similar to the peak of pure berberine (Figure 2(h)). At excitation $488 \mathrm{~nm}$ the shift of the berberine emission to long-wavelength region occurred. Unlike the surface secretory cells and secretion, the pulp of the fruit (Figure 2(e)) had weak emission, while secretory hair (Figure $2(\mathrm{f})$ ) seen on the surface, outside the fruit skin, was lightening in blue. Their fluorescence spectra are represented in Figure 2(g). The secretory hair had maximum $460 \mathrm{~nm}$ (Figure 2(f)), perhaps, belonging to flavonols [2]. Besides the fruits, flowers of $B$. vulgaris were analyzed as possible perspective pharmaceutical material. One can see stereomicroscopy of the tissue in Figures 2(i) and 2(j). Microspectrofluorimeter recorded the fluorescence spectra of main lightening parts of the flower, pistil and pollen (Figure 2(k)). Among the flower parts the stigma of pistil had maximum at $540 \mathrm{~nm}$ like berberine, but pollen peak is $510 \mathrm{~nm}$ that may show the flavin 

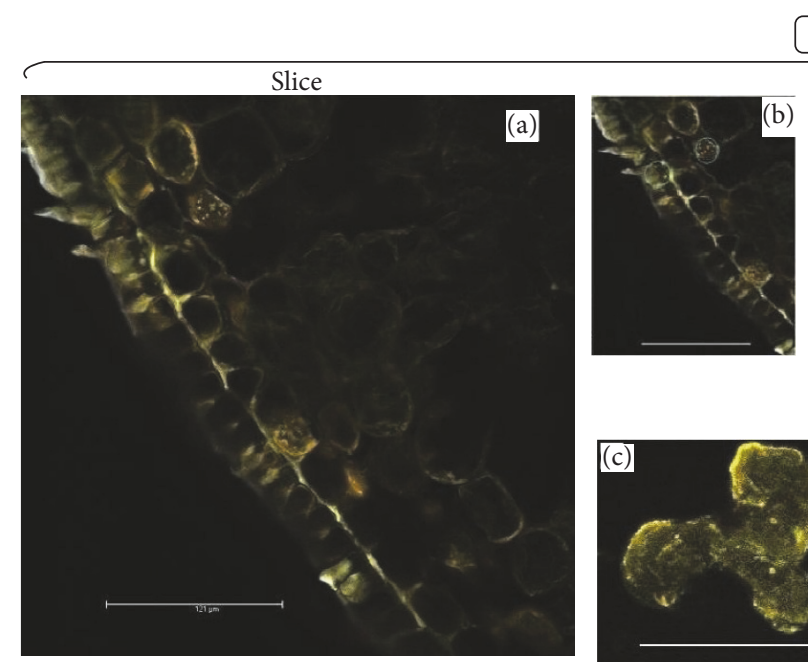

Fruit
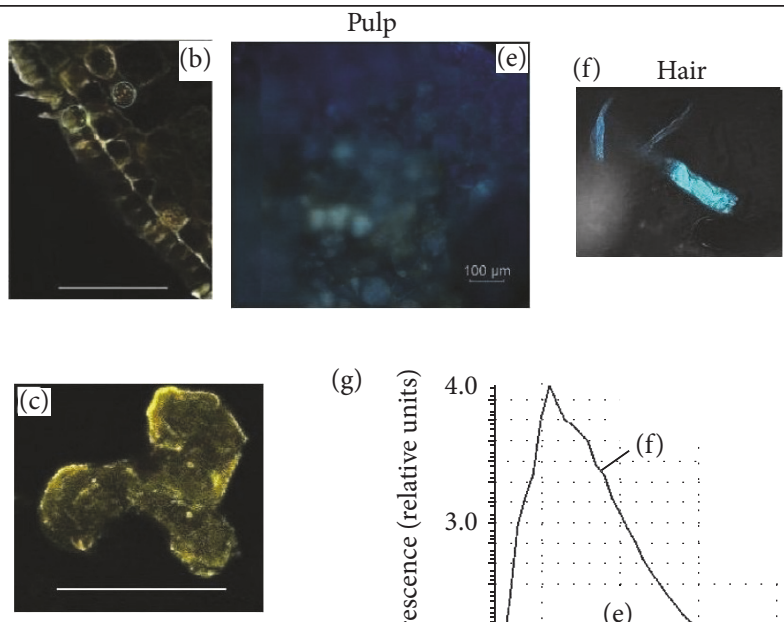

(d)
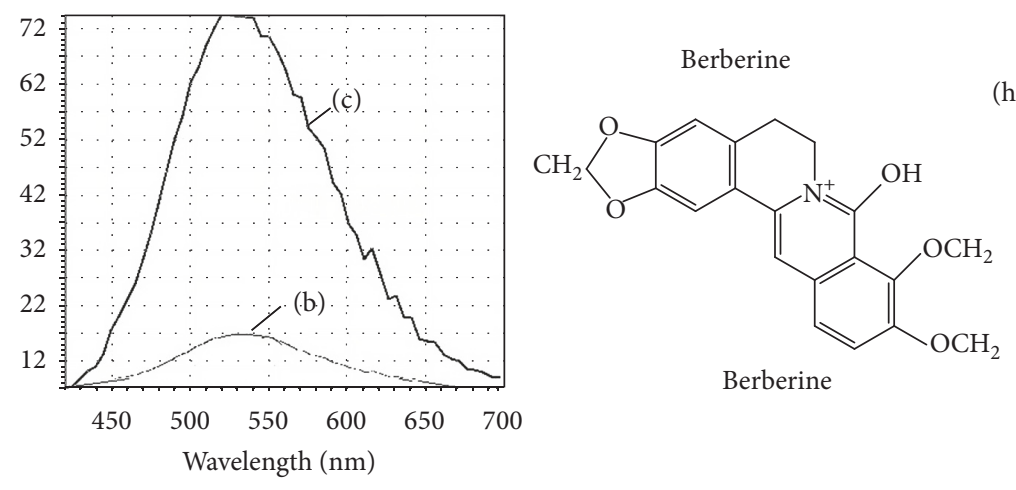

(g)

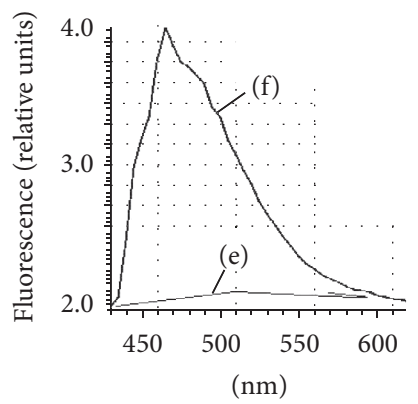

(h)

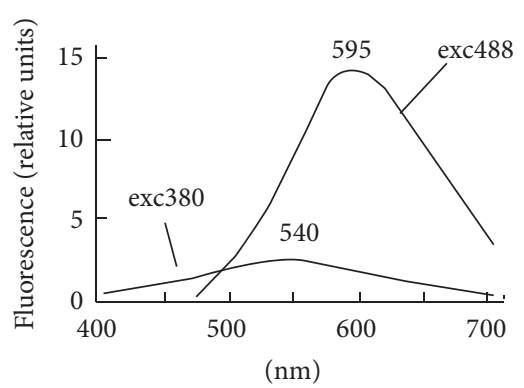

Flower

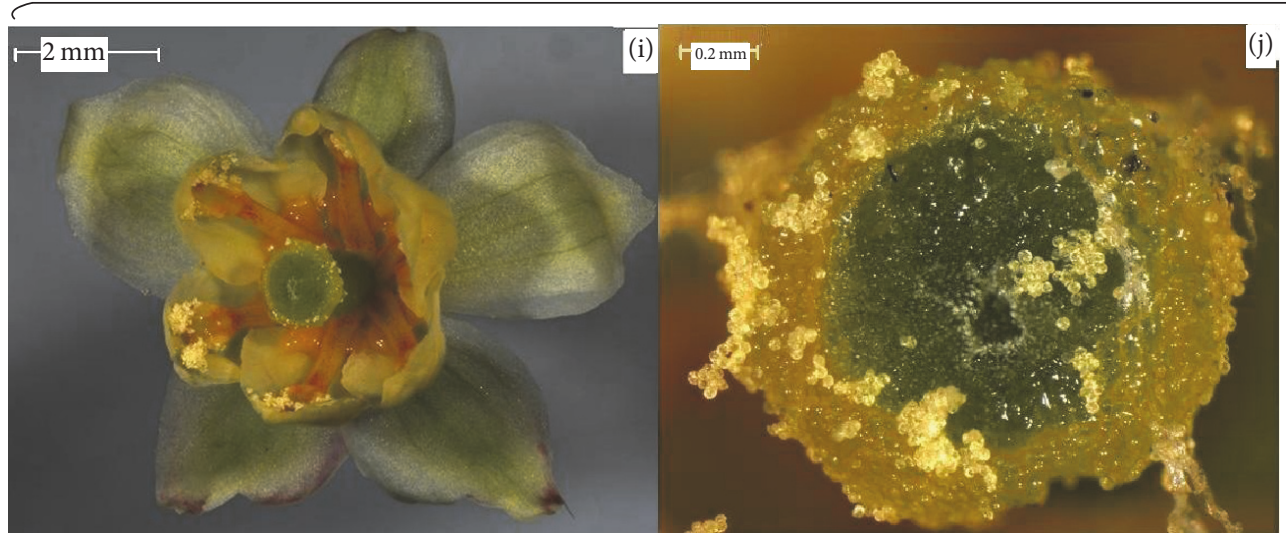

$(\mathrm{k})$

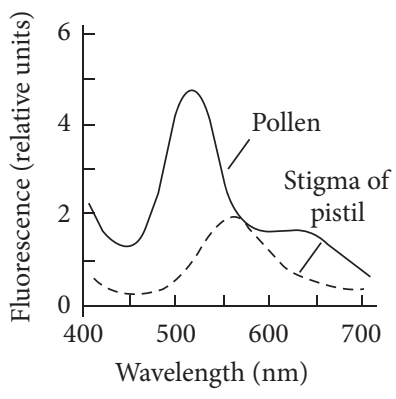

Figure 2: The fluorescence of alkaloid-containing secretory cells from Berberis vulgaris L. Fruit fluorescing under laser-scanning confocal microscope Leica TCS SP-5 (laser excitation $405 \mathrm{~nm})$ : ((a) and (b)) single cells of fruit border on transverse slice, bar $=30 \mu \mathrm{m}$, (c) excretion from the fruit, (d) the fluorescence spectra from (b) (cells emitted in green-yellow are marked by rings) and (c) images, (e) the fruit pulp, (f) secretory hair, (g) the fluorescence spectra from images (e) and (f), and (h) the fluorescence spectra registered by spectrofluorometer Perkin Elmer 350 MPF-44B in $0.5 \mathrm{~cm}^{3}$ cuvette from water solution of $10^{-5} \mathrm{M}$ berberine. Flower: ((i) and (j)) images of whole flower and pistil with pollen on the pistil stigma done by stereomicroscopy and $(\mathrm{k})$ the fluorescence spectra of pollen and pistil recorded by microspectrofluorimetry.

characteristics [2]. Of course, we supposed that other components such as alkaloids columbamine and berbamine [11] also can contribute to the emission, but it needs additional studies with these individual compounds.
Another example of prevailing components in pharmaceutical samples was the bract multicellular glands of matured female flowers (female cones) from hop Humulus lupulus L, which served as a natural sedative drug to calm the nerves, 
reduce stress and anxiety, or even give anticancer effects [10, $11,17,18]$. Active matters in hops are bitter acids humulone and lupulone, weak acid components. A phytoestrogen 8prenylnaringenin has been recently discovered in the female flowers of hops plants [17]. In many cases, extracts of the $H$. lupulus cones may also act as a prooxidants due to the presence of flavonoids $[18,19]$ and have antitumor potential [20]. All active hop components are accumulated in the multicellular glands (our stereomicroscopic images in Figures $3(a)-3(c)$ ) that, maturing, release small glandular cells named as lupulin in pharmacy (Figures 3(b) and 3(c)). The single glandular cells had nuclei seen after the staining by Hoechst 33342 , fluorescent dye for the DNA. For therapy the dried lupulin powder is used. About the autofluorescence of lupulin we had no information earlier. In our experiments one can see blue fluorescence of the multicellular gland under ultraviolet excitation of luminescence microscope (Figure 3(d)) and the emission spectra with maxima $510-520 \mathrm{~nm}$ recorded by microspectrofluorimetry (Figure 3(e)). Confocal microscopy of lupulin cells (Figures 3(f) and 3(g) marked as ROI on the photo) showed the same peak. Bitter acids humulone and lupulone separated on silica gel chromatographic plates in UV-light were emitted in blue [4]. In our experiments bitter acids-enriched water extract from hops glands $(0.5 \mathrm{mg} / 5 \mathrm{ml})$ was emitted with maxima $475-480 \mathrm{~nm}$ (Figure $3(\mathrm{~h})$ ). Bitter acids, flavonoids (that fluoresce at $460-480 \mathrm{~nm}$ ) and the phenol estrogen, perhaps, contribute to the emission.

Third example of prevailing components in pharmaceutical material which has been studied was wild camomile or chamomile (Matricaria chamomilla L.), a well-known medicinal plant species from the Asteraceae family. Nowadays it is a highly favored and much used medicinal plant in folk and traditional medicine $[10,11,21]$ due to specific aroma components and constituents of essential oil from leaves and flowers. The plant species has multitherapeutic, cosmetic, and nutritional values. It is often used as natural antiinflammatory, antibacterial, and spasmolytic drug $[10,11]$. Moreover, the chamomile azulenes and proazulenes found in essential oil demonstrated antiallergic features [11]. Some phenols and monoterpenes are included in the emission but in smaller degree in the glands and mainly concentrated in secretory hairs $[10,11]$. In our work fluorescent analysis of fresh herbal material, leaf and flower from $M$. chamomilla, is shown in Figure 4. Glandular hairs of the leaf that stored valuable pharmaceuticals have been demonstrated to fluoresce, mainly, in blue or green channels of confocal microscope (Figure 4(a)) at excitation by laser $405 \mathrm{~nm}$, while in red channel of the apparatus chlorophyll was seen (Figure 4(b)). Largest lightening was observed in the base part of the trichome. In the emission spectra from the parts of the hairs fluorescing in blue-green big maxima $500-510 \mathrm{~nm}$ was seen, while small peak $680 \mathrm{~nm}$ belonged to chlorophyll (Figure 4(a)). In red channel the green pigment emission prevailed in the spectrum (Figure 4(b)). On optical slices via $1 \mu \mathrm{m}$ (Figure $4(\mathrm{c})$ ) one can see the spreading of blue-fluorescing secretion along the multicellular hair fulfills various fluorescing products. The emission may belong to azulenes or/and flavonoids prevailing in M. chamomilla [11, 21]. Isolated azulene chamazulene [22] from the species was emitted at $425-430 \mathrm{~nm}$ [1], while most of flavonoids were at $460-490 \mathrm{~nm}$ [2]. Pollen and petals from the ligulate flowers of M. chamomilla also were emitted in blue-green (Figures 4(d) and 4(e)), perhaps, also due to the flavonoids and azulene content. The spikes of pollen grains appeared as brighter lightening parts, than other surfaces (Figure 4(d)). As seen in fluorescence spectra of Figures 4(e) and 4(f) (spectra 1-5) the composition of compounds in the emitted petal and pollen varied, from blue-green to yellow, which showed the contribution of flavins (at 520-530 nm, mainly, in petal gland spectrum number 1), carotenoids (530-540 nm, especially in pollen grains), coumarins $(500-540 \mathrm{~nm})$, and some flavonoids (460-480, 580-590 nm) [2]. Unlike the leaf, in the petals there was no chlorophyll in the glands seen from image spectrum (f). However, it should mark that azulenes of the pollen may be bounded with cell walls and, in this case, the pigments give also a contribution to red emission (maximum $620 \mathrm{~nm}$ ) [23]. According to the fluorescence spectra recorded earlier by microspectrofluorimetry [2] pollen of chamomile may have maxima $435,465,570$, and $620 \mathrm{~nm}$, with glands of ligulate flower having 460,550 , and $650 \mathrm{~nm}$. The spectra differed from the leaf glandular hairs because of lack of chlorophyll maximum $680 \mathrm{~nm}$. Glandular structures of the chamomile leaf fluoresced with maxima 460, 550, and $675 \mathrm{~nm}$ $[1,2]$. Thus, the use of microspectrofluorimetry gave more peaks in the fluorescence spectra, than our measurement by confocal microscopy.

3.3. Perspectives and Difficulties. Based on the autofluorescence as a parameter for the characteristics of raw pharmaceutical material one may carry out the analysis to show the following: (1) concrete secretory structures fulfilling drug components in vivo without any biochemical procedures and tissue disruption; (2) localization of some medicinal drugs, secondary metabolites, in intact cells, organs, and tissues; (3) express-identification of prevailing compound in the sample if possible. Along with the visible preferences in the fluorescent analysis of intact pharmaceutical material we need to mark a limitation and additional factors influencing on the determinations as well. First of all it is the interference of the wavelengths by some phenols and terpenoids that fluoresces in blue $[2,7]$. It may be overcome via the extraction of the components by hydrophilic and hydrophobic solvents and then the record of the fluorescence spectra, like what has been described earlier $[2,24]$. The disappearance or shift of maxima in the fluorescence spectra after similar procedures shows that some extracted compounds may contribute to the emission of intact cells, like what was earlier demonstrated for pollens $[2,24]$. In complex mixture of natural composition a weak fluorescence, peculiar to one component, may be masked by bright emission of another component in the same spectral region too. In this case, the extraction of the masking components by hydrophilic or hydrophobic solvents is also needed in order to compare the fluorescence spectra of the samples before and after the appropriate extraction procedure. The changes in the position of maxima in the emission spectra also depend on the stage of plant development; for example, the developing glands of Humulus 

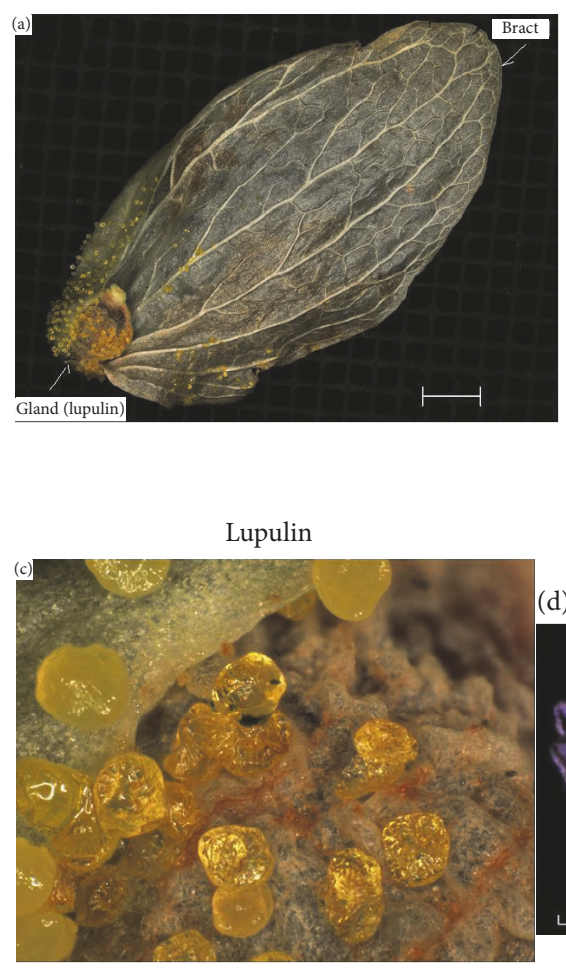

(d)

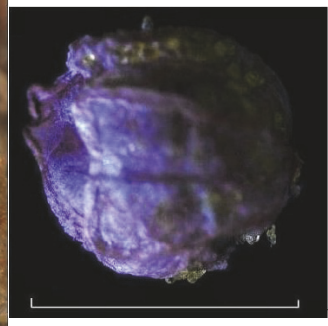

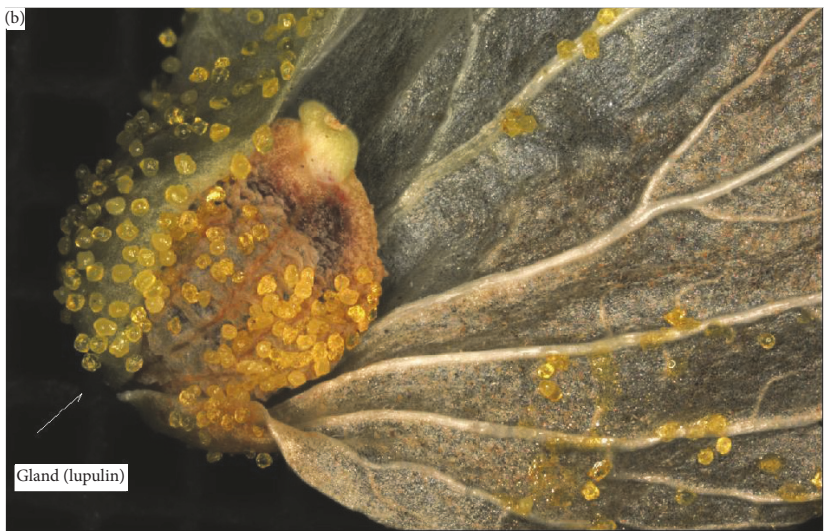

(e)
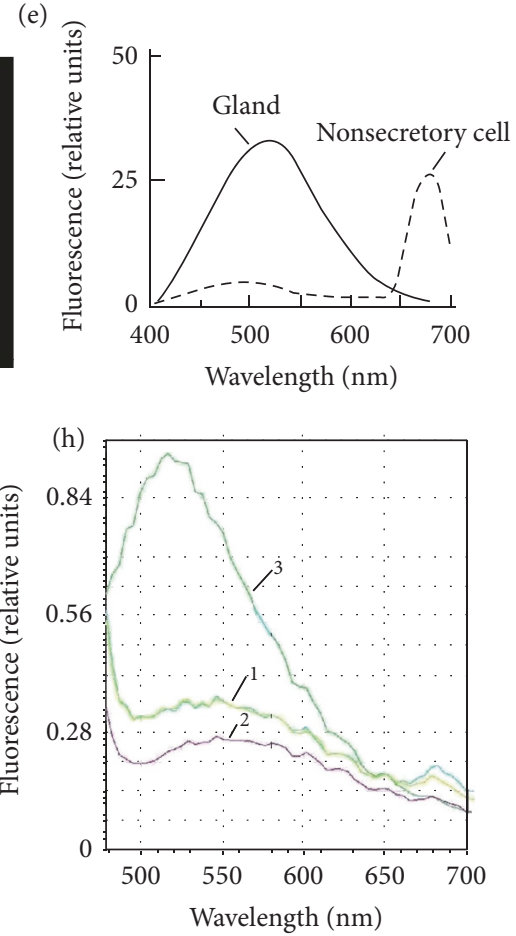
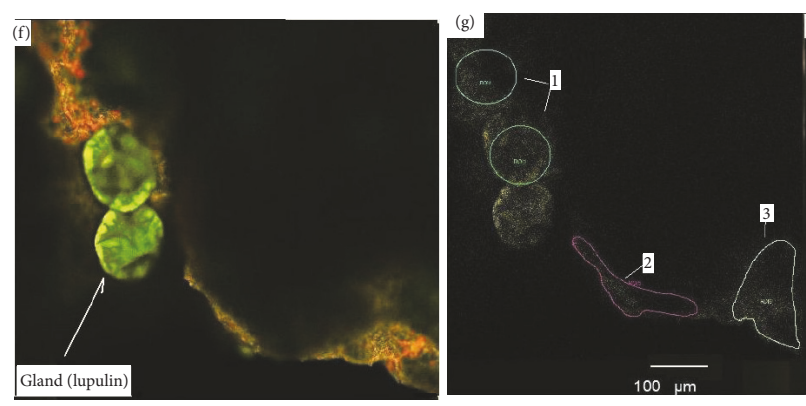

(i)<smiles>CC(C)CCC1C(O)C(CC(C)C)C(O)C(O)(CC(C)C)C1O</smiles>

Humulone

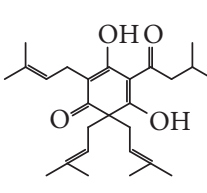

Lupulone

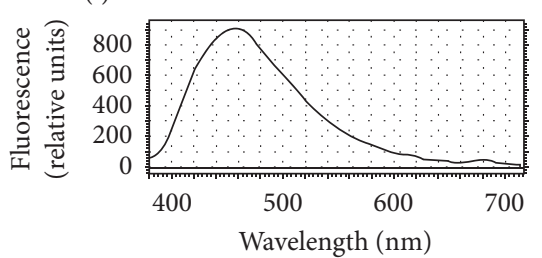

Figure 3: The fluorescence of pharmaceutical valuable secretory cells of female flower cone bracts from Humulus lupulus L. ((a), (b), (c)) Stereomicroscopic images of multicellular resinous glands which single cells are liberated and named as lupulin; (d) fluorescence of multicellular gland under UV-light of luminescence microscope Leica D $6000 \mathrm{~B}$; (e) the fluorescence spectra of the gland recorded by microspectrofluorimeter MSF-1; (f) common image of glandular surface fluorescing under laser-scanning confocal microscope Leica TCS SP-5 (laser excitation $405 \mathrm{~nm}$ ) in green-yellow and (g) ROI of the image (marked by blue and violet rings, numbers 1, 2, and 3, bar $=100 \mu \mathrm{m}$ ); (h) the fluorescence spectra of the ROI marked as 1,2, and 3 on image (g); (i) the fluorescence spectra of water bitter acid-containing extract from glands $(1: 10 \mathrm{w} / \mathrm{v})$ registered by spectrofluorometer Perkin Elmer $350 \mathrm{MPF}-44 \mathrm{~B}$ in $0.5 \mathrm{~cm}^{3}$ cuvette, excitation $360 \mathrm{~nm}$.

lupulus cones mainly fluoresce in blue, while matured ones fluoresce in green [2].

Differences in the position of the emission maxima for one and same plant species appear to depend on the media of secretory cells, which may additionally contain oil, phenols, oleophenols, oleoresins, and others in combinations found in the cells [2]. Here it is likely to use modeling between main fluorescent component analyzed and possible medium via 


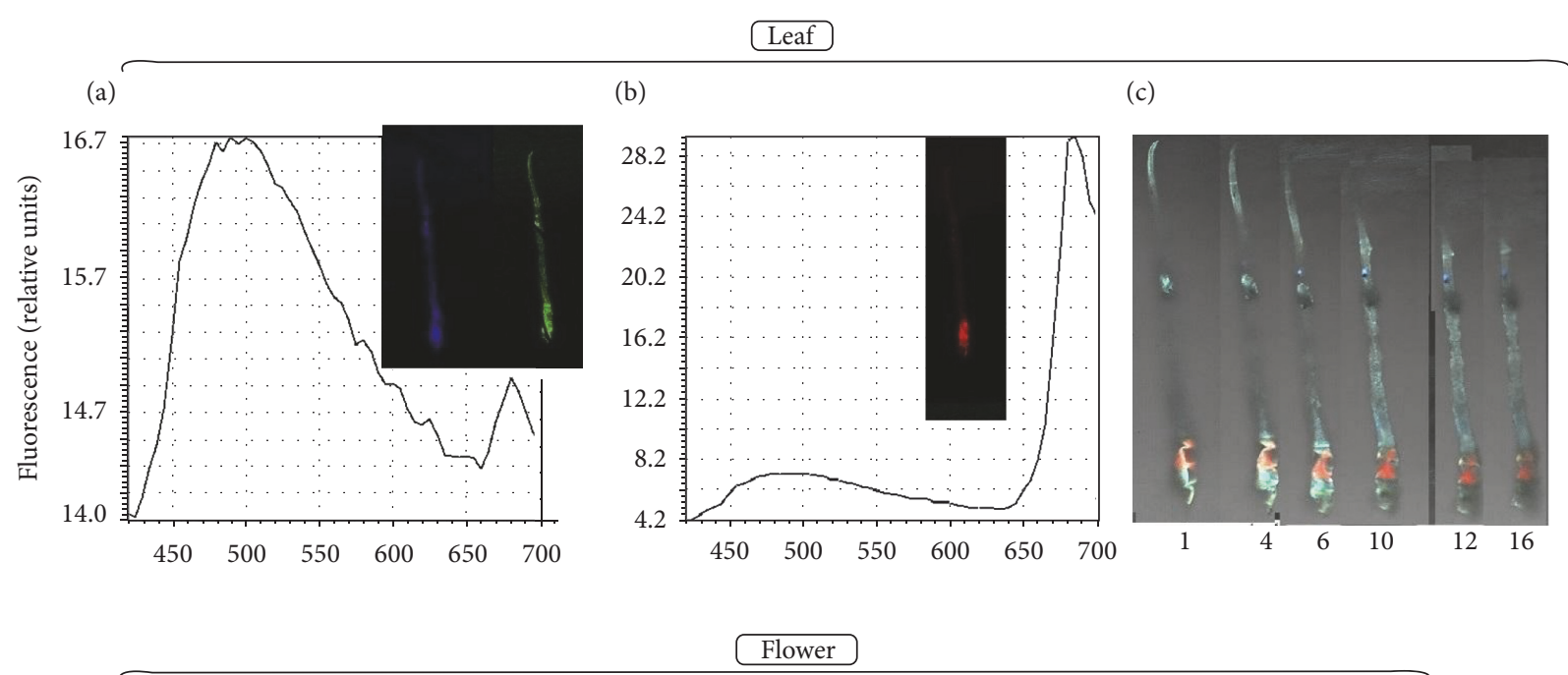

(d)

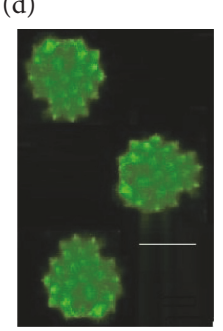

Pollen (e)

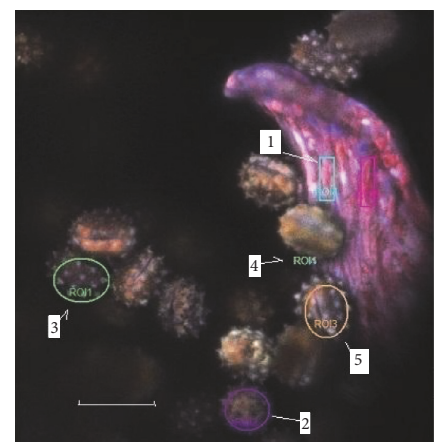

(f)

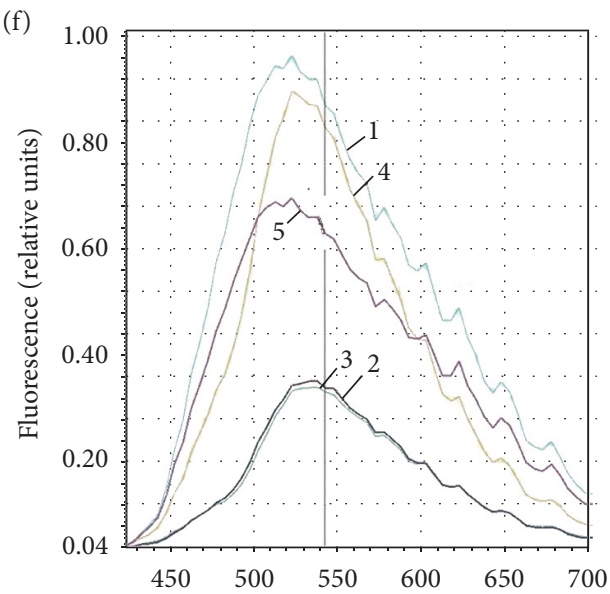

FiguRE 4: The fluorescence of terpene-containing secretory cells of hairs from Matricaria chamomilla L. Leaf. ((a) and (b)) Images of the hair in three channels of laser-scanning confocal microscope Leica TCS SP-5 (laser excitation $405 \mathrm{~nm}$ ) and (c) longitudinal hair optical slices. Flower: (d) image of pollens from flower made by laser-scanning confocal microscope Karl-Zeiss (laser excitation $488 \mathrm{~nm}$ ); (e) image of the part from ligulate white flower with pollen recorded by laser-scanning confocal microscope Leica TCS SP-5 (laser excitation $405 \mathrm{~nm}$ ) and its ROI marked by rings (1-5), bar $=30 \mu \mathrm{m}$; (f) the fluorescence spectra of the marked ROI (1-5).

a preparation of mixtures between the compound analyzed and other components of the medium studied. After that one could record their fluorescence spectra in order to determine true maximum and shifts $[2,24]$. For example, crystal of phenol rutin is lightening with maximum $620 \mathrm{~nm}$, but in the mixture with menthol oil the peak is $480-485 \mathrm{~nm}$, while crystals of alkaloid rutacridone that fluoresce with main maxima 595-600 $\mathrm{nm}$ stored the same peak in similar mixture [2]. All this should be subject of the attention in future investigations.

\section{Conclusion}

Autofluorescence has perspectives as one of the parameters of the express-analysis of pharmaceutical materials. The location of secretory cells containing natural drugs and the accumulation of secondary metabolites in the structures may be observed with luminescence microscopy and its recording modifications such as microspectrofluorimetry and confocal microscopy. Secretory cells of plants contain various fluorescing pharmaceuticals such as alkaloids, terpenes, phenols, flavins, and quinones, and their emission differs from chlorophyll. Based on the emission characteristics (color images and the fluorescence spectra) scientist could discriminate secretory cells from nonsecretory ones as well as identify prevailing component of the secretions. The luminescence in the express-analysis opens perspectives to know fast whether the plant material is ready or not for pharmacy.

\section{Conflicts of Interest}

The authors declare that they have no conflicts of interest.

\section{References}

[1] V. V. Roshchina, "Autofluorescence of plant secreting cells as a biosensor and bioindicator reaction," Journal of Fluorescence, vol. 13, no. 5, pp. 403-418, 2003.

[2] V. V. Roshchina, Fluorescing World of Plant Secreting Cells, Science, Enfield, Plymouth, UK, 2008. 
[3] V. V. Roshchina, A. V. Yashina, V. A. Yashin, and M. V. Goltyaev, "Fluorescence of biologically active compounds in plant secretory cells," in Forestry and Agroforestry, S. S. Narwal, P. Pavlovic, and., and J. John, Eds., vol. 2, pp. 3-25, Studium Press, Houston, Tex, USA, 2011.

[4] H. Wagner and S. Bladt, Plant Drug Analysis: A Thin Layer Chromatography Atlas, Springer, Berlin, Heidelberg, 1996.

[5] R. Upton, A. F. Graff, G. Joliffe, R. Linger, and E. M. Williamson, Eds., Microscopic Characterization of Botanical Medicines, CRC Press, Boca Raton, Fla, USA, 2011.

[6] V. V. Roshchina, "Vital autofluorescence: application to the study of plant living cells," International Journal of Spectroscopy, vol. 2012, pp. 1-14, 2012.

[7] V. V. Roshchina, A. V. Kuchin, and V. A. Yashin., "Autofluorescence of plant secretory cells as possible tool for pharmacy," International Journal of Pharmacy and Chemistry, vol. 2, no. 2, pp. 31-38, 2016.

[8] V. V. Roshchina, E. V. Melnikova, V. N. Karnaukhov, and B. N. Golovkin, "Application of microspectrofluorimetry in spectral analysis of plant secretory cells," Biological Bull no 2, pp. 167171, 1997.

[9] V. N. Karnaukhov, V. A. Yashin, V. I. Kulakov, V. M Vershinin, and V. V Dudarev, Apparatus for investigation of fluorescence characteristics of microscopic objects', US Patent, N 4, 354, 114 : $1-14,1982$.

[10] J. A. Duke, Handbook of Medicinal Herbs, CRC Press, Boca Raton, Fla, USA, 2002.

[11] D. A. Murav'eva, I. A. Samylina, and P. Yakovlev, Pharmacognosy, Meditsina, Moscow, 2007.

[12] C. S. C. Garcia, C. Menti, A. P. F. Lambert et al., "Pharmacological perspectives from brazilian Salvia officinalis (Lamiaceae): Antioxidant, and antitumor in mammalian cells," Anais da Academia Brasileira de Ciências, vol. 88, no. 1, pp. 281-292, 2016.

[13] S. Jantová, R. Hudec, S. Sekretár, J. Kučerák, and M. Melušová, "Salvia officinalis L. extract and its new food antioxidant formulations induce apoptosis through mitochondrial/caspase pathway in leukemia L1210 cells," Interdisciplinary Toxicology, vol. 7, no. 3, pp. 146-153, 2014.

[14] M. Hamidpour, R. Hamidpour, S. Hamidpour, and M. Shahlari, "Chemistry, pharmacology, and medicinal property of sage (salvia) to prevent and cure illnesses such as obesity, diabetes, depression, dementia, lupus, autism, heart disease, and cancer," Journal of Traditional and Complementary Medicine, vol. 4, no. 2, pp. 82-88, 2014.

[15] M. Wink, "Modes of Action of Herbal Medicines and Plant Secondary Metabolites," Medicines, vol. 2, no. 3, pp. 251-286, 2015.

[16] M. Rahimi-Madiseh, Z. Lorigoini, H. Zamani-gharaghoshi, and M. Rafieian-kopaei, "Berberis vulgaris specifications and traditional-uses," Iranian Journal of Basic Medical Sciences, vol. 20, no. 5, pp. 569-587, 2017.

[17] L. R. Chadwick, G. F. Pauli, and N. R. Farnsworth, "The pharmacognosy of Humulus lupulus L. (hops) with an emphasis on estrogenic properties," Phytomedicine, vol. 13, no. 1-2, pp. 119131, 2006.

[18] J. Olšovská, V. Bostikova, M. Dušek et al., "Humuluslupulus L (hops)- a valuable source of compounds with bioactive effects for future therapies," Military Medical Science Letters, vol. 85, no. 1, pp. 19-30, 2016.

[19] M. Karabin, T. Hudcova, L. Jelinek, and P. Dostalek, "Biotransformations and biological activities of hop flavonoids," Biotechnology Advances, vol. 33, no. 6, pp. 1063-1090, 2014.
[20] E. F. Fang and T. B. Ng, Antitumor Potential and other Emerging Medicinal Properties of Natural Compounds, Springer, Heidelberg, Germany, 2013.

[21] O. Singh, Z. Khanam, N. Misra, and M. K. Srivastava, "Chamomile (Matricaria chamomilla L.): an overview," Pharmacognosy Reviews, vol. 5, no. 9, pp. 82-95, 2011.

[22] V. V. Roshchina and V. D. Roshchina, The Excretory Function of Higher Plants, Springer Berlin Heidelberg, Berlin, Heidelberg, 1993.

[23] V. V. Roshchina, E. V. Melnikova, N. A. Spiridonov, and L. V. Kovaleva, "Azulenes, the blue pigments of pollen," Doklady Biological Sciences, vol. 340, pp. 93-96, 1995.

[24] E. V. Melnikova, V. V. Roshchina, and V. N. Karnaukhov, "Microspectrofluorimetry of plant pollen," Biophysics, vol. 42, pp. 226-233, 1997. 

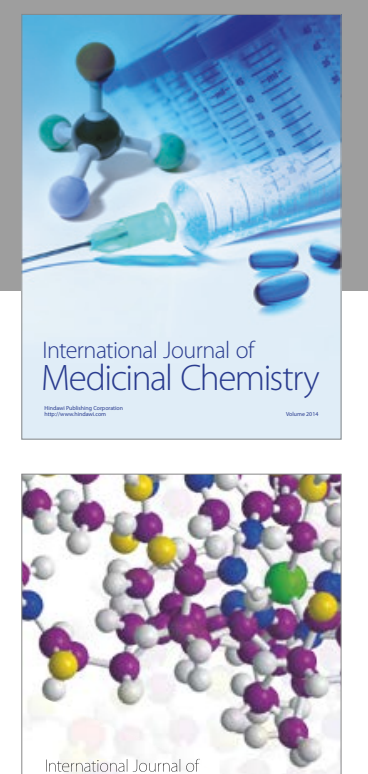

Carbohydrate Chemistry

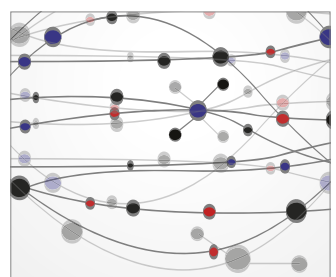

The Scientific World Journal
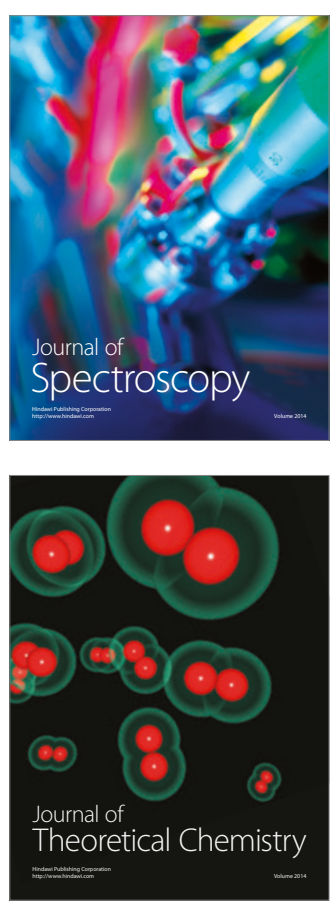
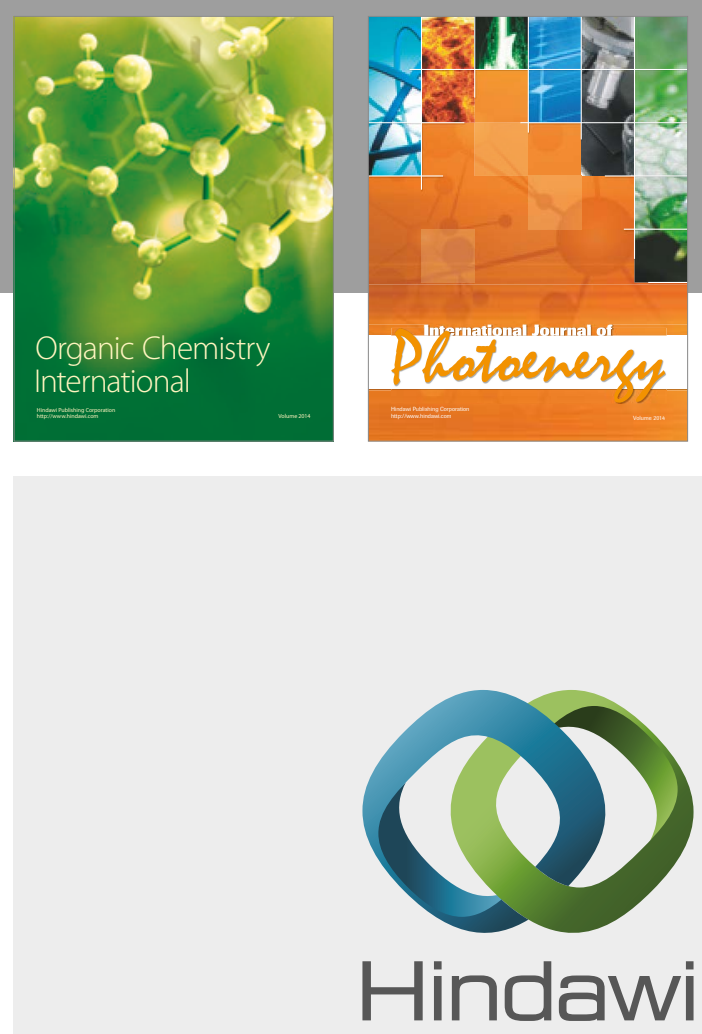

Submit your manuscripts at

https://www.hindawi.com

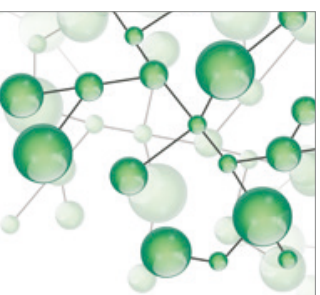

International Journal of

Inorganic Chemistry

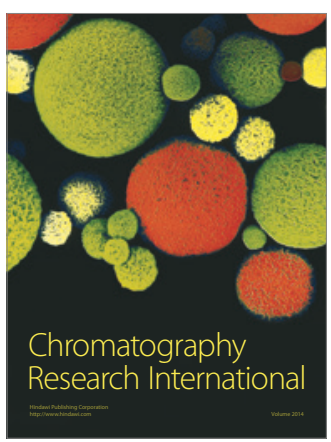

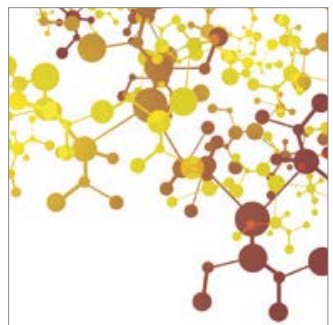

Applied Chemistry
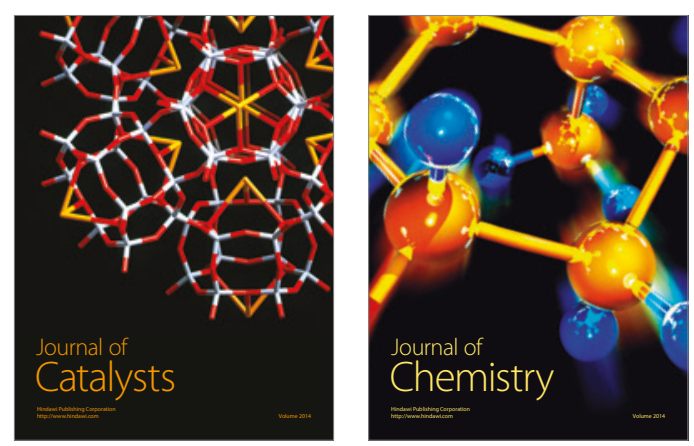
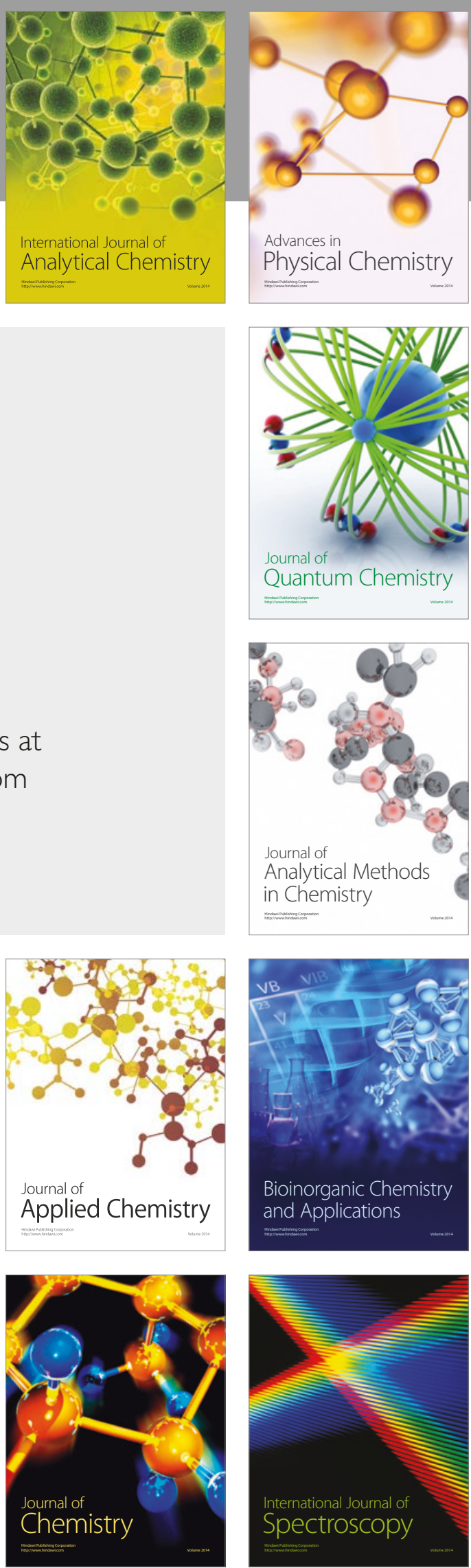